\title{
Héroïnes et mémoire familiale dans le Chevalier errant de Thomas de Saluces
}

Female heroes and familial memory in Thomas de Saluces's le Chevalier errant

\section{Florence Bouchet}

\section{Q OpenEdition}

1 Journals

Édition électronique

URL : http://journals.openedition.org/clio/9420

DOI : 10.4000/clio.9420

ISSN : 1777-5299

Éditeur

Belin

Édition imprimée

Date de publication : 15 décembre 2009

Pagination : 119-136

ISSN : $1252-7017$

\section{Référence électronique}

Florence Bouchet, « Hérö̈nes et mémoire familiale dans le Chevalier errant de Thomas de Saluces »,

Clio. Histoire, femmes et sociétés [En ligne], 30 | 2009, mis en ligne le 15 décembre 2012, consulté le 19 avril 2019. URL : http://journals.openedition.org/clio/9420 ; DOI : 10.4000/clio.9420 


\section{Regards complémentaires}

\section{Héroïnes et mémoire familiale dans le Chevalier errant de Thomas de Saluces}

Florence BOUCHET

Si le mot «héros » est employé dans les années 1370 par Nicole Oresme ${ }^{1}$, il a fallu attendre la deuxième moitié du XVI $\mathrm{XI}^{\mathrm{e}}$ siècle pour voir paraître « héroïne » sous la plume de Ronsard. Ajoutons à cela la misogynie foncière des clercs médiévaux, qui s'est autorisée de poètes antiques $^{2}$, de philosophes et des Pères de l'Église ${ }^{3} .$. et la possibilité d'existence d'une quelconque héroïne au Moyen Âge devient un sujet d'inquiétude. Plus largement, selon Marc Tourret,

Il semble que l'énumération des grandes figures héroïques occidentales consiste à dresser la liste de membres d'un club essentiellement masculin s'autorisant à accomplir des « exploits » pour réaffirmer, par la maittrise de la violence, un ordre sexuel inégal et androcratique. L'accès des femmes à l'héroïsme est d'autant plus problématique que les activités du héros étaient traditionnellement masculines (la guerre, l'exploration du monde) et que la célébrité qui y était associée était plutôt réservée aux hommes ${ }^{4}$.

\footnotetext{
C'est un mot savant, qui restera rare jusqu'au XVII $\mathrm{s}$.

Notamment les Remedia amoris d'Ovide et les Satires de Juvénal.

3 Le traité Adversus Jovinianum de Théophraste, meilleur disciple d'Aristote, s'est perdu mais a été partiellement transmis par saint Jérôme.

$4 \quad$ Faliu \& Tourret $2007: 218$.
} 
La lecture des textes médiévaux amène toutefois à réviser cette vision androcentrée de l'hérö̈sme. Je m’intéresserai ici au Livre du Chevalier errant du marquis Thomas III de Saluces ${ }^{5}$, sorte de roman d'apprentissage allégorico-encyclopédique qui relate les aventures d'un Chevalier (figure spéculaire de Thomas) confronté successivement au dieu d'Amour, à Dame Fortune et à Dame Connaissance. Les circonstances de la rédaction orientent la visée politique de l'œuvre: Thomas a entamé la composition de son roman entre 1394 et 1396, alors qu'il était prisonnier d'un allié du comte de Savoie Amédée VIII qui lui disputait ses terres. Il s'agissait donc, pour notre marquis, de conforter son pouvoir menacé en réaffirmant sa légitimité et l'ancienneté de sa noble lignée ${ }^{6}$. Mêlant aux souvenirs d'ordre historique les séductions de la légende et de l'imaginaire, le Livre du Chevalier errant fait office de "défense et illustration» de la mémoire familiale des Saluces. Bien que les femmes jouent un rôle déterminant dans les alliances politico-matrimoniales en vue de conforter le pouvoir des familles princières, elles sont très minoritaires dans les généalogies médiévales ${ }^{7}$. Lorsqu'elles y figurent, c'est qu'elles ont un rôle particulier à jouer, comme on va le vérifier à travers quelques figures saillantes de la galerie familiale élaborée par Thomas de Saluces ${ }^{8}$.

\section{Un vaste roman familial}

Tout comme les Nations ont eu besoin, à l'époque moderne, de héros pour forger leur identité nationale ${ }^{9}$, les familles médiévales de haut rang ont eu besoin de héros identificatoires. La structure

5 Saluces : cité du Piémont italien (à une cinquantaine de kilomètres au sud de Turin), capitale d'un marquisat fondé en 1142.

6 Cette pratique était déjà ancienne: Duby 1988 a analysé la littérature généalogique française des $\mathrm{XI}^{\mathrm{e}}-\mathrm{XII}{ }^{\mathrm{e}}$ siècles qui semblait «répondre au souci de légitimer un pouvoir » (p. 172).

7 Butaud \& Piétri 2006 : 217-218.

8 Le développement qui suit s'appuie sur des analyses amorcées dans ma thèse de doctorat non publiée : Lire, voir, écrire au XIVe siècle : étude du Livre du Chevalier errant de Thomas de Saluces. Robert Fajen 2003 s'est par la suite intéressé aux implications familiales du Livre du Chevalier errant dans sa propre thèse.

9 Centlivres, Fabre \& Zonabend 1998. 
narrative du Livre du Chevalier errant favorise l'insertion d'allusions, d'anecdotes et de récits plus développés ${ }^{10}$ qui reconstituent par fragments les cinq générations qui ont précédé Thomas III sur le trône de Saluces, à partir de Thomas I ${ }^{e r}$ (soit un siècle et demi environ d'histoire familiale) ${ }^{11}$; au-delà, la mémoire historique est relayée par une mémoire légendaire. Thomas conclut fièrement son ouvrage en affirmant :

Ma mère fu de Geneve de la noble ligné

Le fort Olivier, le noble combattant,

Qui fu preuz en armez et fu cremuz* tant, *

Qui conquist Fierebraz, le cremuz payn*, *païen

Qui aux gens Charlemaine menoit bel hutin*. (v. 10321-25) ${ }^{12} \quad$ *combat

Thomas de Saluces s'est fabriqué cette ascendance prestigieuse qui apparente sa mère au preux Olivier immortalisé par les chansons de geste, en manipulant le lignage de Garin de Monglane ${ }^{13}$. Par cette «invention d'ancêtre épiques", il rejoint «le souci nouveau [au XII siècle] de pousser le souvenir ancestral jusqu'au cœur de l'époque carolingienne, c'est-à-dire jusqu'au moment privilégié décrit par les chansons de gestes et de dépasser, par conséquent, le seuil chronologique auquel s'arrêtait jadis la mémoire de la parenté $»^{14}$. La

10 Bouchet 2001.

11 Étudiant des textes des XII ${ }^{\mathrm{e}} \mathrm{XIII}{ }^{\mathrm{e}}$ siècles, Guenée 1976-1977 conclut : « indépendamment de l'importance des événements, des hommes et des familles, indépendamment même du milieu de transmission, la tradition orale obéit à des lois qui ne varient guère. Du passé le plus proche, elle retient, sur 60 ou 70 ans, des événements précis qu'elle sait dater. Remontant plus haut, elle conserve assurément la mémoire des faits importants, mais ceux-ci ne sont plus chronologiquement situés. Nous savons, nous, que ces faits peuvent avoir, un peu plus un peu moins, une centaine d'années. Au-delà, c'est la nuit, que percent seuls quelques noms » (p. 34).

12 Citation d'après Ward 1984 ; je supprime la virgule à la fin du v. 10321. Les références (pagination et chapitres) des citations suivantes du Livre du Chevalier errant renvoient à cette même édition.

13 Le chap. 251 dote Renier, l'un des fils de Garin, du fief de Genève et fait d'Olivier son fils et l'héritier de Genève (alors que selon Bertrand de Bar-surAube, dans la chanson de geste Girart de Vienne, c'est Gênes que Renier reçut en fief de Charlemagne).

14 Duby 1988 : 179. 
démarche de généalogiste de Thomas de Saluces peut être mise en relation avec la pratique, de plus en plus fréquente et approfondie aux XIVe-XVe siècles, des « livres de raison » ou « livres de famille », sortes de chroniques domestiques où étaient consignés au fil des générations renseignements généalogiques, histoires et légendes (généralement héritées d'une tradition orale) au sujet de certains membres de la famille, réflexions morales, etc. Ces ricordanze très en faveur en Italie sont particulièrement révélatrices des mentalités familiales (au sens clanique du terme $)^{15}$. Or les femmes, bien que numériquement minoritaires dans ce miroir familial qu'est Le Chevalier errant, se signalent par un relief particulier. Comme l'explique G. Duby, «le fil généalogique en remontant vers le passé peut subir des décrochements, abandonner la lignée patrilinéaire moins illustre pour, à partir de telle aïeule dont les descendants ont conscience de tirer un héritage plus éclatant, remonter de fils en père cette lignée plus honorable $»^{16}$. De plus, dans le projet quelque peu fantasmatique qui est celui de Thomas de Saluces, les femmes ont un rôle particulier à jouer, que suggère M. Pastoureau lorsqu'il affirme que « c'est toujours par les femmes que se transmettent au sein des lignages aristocratiques les emblèmes et les mythes $»^{17}$. Dès lors, il est possible de parler d'héroïnes et je m'attacherai aux deux plus remarquables dans Le Chevalier errant.

\section{Richarde de Saluces, la guerrière}

Il n'est pas nécessaire de remonter bien haut dans la généalogie des Saluces pour y rencontrer une femme sortant du commun: la

15 Ariès \& Duby 1985 : 260. Pour une mise au point récente sur ce genre textuel (relativement polymorphe) et son exploitation historiographique, Mordenti 2004 ; Cazalé Bérard \& Klapisch-Zuber 2004. Le Chevalier errant était donc vraisemblablement d'abord voué à circuler dans le cercle familial, plutôt qu'à faire carrière littéraire. À la fin du manuscrit BnF fr. 12559, longtemps resté possession familiale des Saluces, Louis Ier, fils de Thomas III, fit inscrire les dates de naissance des cinq premiers enfants qu'il eut (entre 1437 et 1442) d'Isabelle de Montferrat, consacrant l'usage du codex comme livre de raison.

16 Duby $1988: 174$.

17 Pastoureau $1986: 149$. 
grand-mère de Thomas III, Richarde, épouse de Thomas II de Saluces $^{18}$ et fille unique de Galeazzo Ir Visconti, le puissant seigneur de Milan ${ }^{19}$. Dame Connaissance (locuteur digne de foi s'il en est) raconte au Chevalier comment, alors que son époux avait été fait prisonnier par ses ennemis, elle a obtenu la libération de son mari et sauvé son territoire. Délaissant toute langueur féminine (« elle vit bien que plaindre ne souspirer ne lui valoit fors que perdre temps $»^{20}$ ), elle part chercher des secours en Lombardie et retourne vite se battre :

Elle mena ses gens aux frontières et la les ordonna avec ses gens et commença a faire la greigneur guerre du monde. Celle dame, avec ses nobles, ordonnoit avec le cappitain[e] dessus nommez, les coureries et les emprises de grever ses ennemiz. Et quant il y avoit aucune grosse besoingne a faire, elle y vouloit estre et aller, et ja n'eust souffert que autre bannière y feust que la sienne $[. .$.$] . Si devez savoir que toudiz elle$ s'armoit bien et honnourablement, et si faisoit sa lance porter après soy ainsi comme un capitaine, et son harnois de teste et toudis son baston en sa main a ordonner ses genz, et avoit ses dames et damoiselles ordonnées avec elle en ceste besoingne. Et quant venoit caz d'aucunez forteressez a ordonner secours et a les assegier, elle faisoit ce que son mari eust fait et si diligemment que a painez se pourroit croire. [...] par son senz et travail, elle pourchaça tant et tellement que premierement rienz ne fu depuis perduz de sa terre, secondement que ses ennemis moult grevoit et tellement que a la fin ilz mistrent le marquiz a telle finance que bien lui plot, et le trest de la prison [...]. (p. 1049-50)

Lorsque son époux libéré met le siège autour de Saluces, c'est encore elle qui part chercher auprès du marquis de Montferrat, parent des Saluces, d'indispensables renforts militaires ${ }^{21}$. Douée d'initiative, intrépide, Richarde est explicitement haussée à l'égal d'un héros masculin. Si la virtus, ensemble des qualités morales et physiques susceptibles de caractériser un héros, semblait étymologiquement réservée au masculin (vir), l'exemple de Richarde démontre qu'une femme forte peut y prétendre - à condition, toutefois, de renoncer à

18 Il régna de 1336 à 1357.

19 De 1322 à 1328. Et Connaissance de préciser que si Richarde « eust esté un filz, elle eust eu la seingnorie de Milan » (chap. 350, p. 1048).

20 Ward $1984: 1048$.

21 Thomas III, durant sa captivité, rêvait sans doute que sa propre épouse, Marguerite de Roucy, fût capable de la même vaillance ! 
sa féminité pour devenir précisément une virago, une hérö̈ne épique ${ }^{22}$ dont la valeur mériterait une consécration littéraire (c'est justement ce qu'esquisse Thomas de Saluces) :

Assez, beau filz, te pourroie [je] dire de ceste vaillant[e] contesse, et de ses fais et senz on pourroit faire un grant livre, mais je n'en ay guerez raconté de ses fais, fors seulement un petit, pour la memoire que de elle ay pour sa grant vaillance. (p. 1050-51)

Les «fais » sont bien les actions d'éclat, les gesta qui président à la chanson de geste; couplés au «senz», ils réactualisent les valeurs complémentaires de la sapientia et de la fortitudo dans la tradition épique. Connaissance loue au Chevalier sa grand-mère qui «si vaillant et de hault cuer et de bonne renommée fu » (p. 1045) et conclut, au terme d'un récit qui a bien su alternativement jouer de l'hyperbole et de la litote, en la lui donnant en exemple :

Or t'ay filz, dit qui fu ta grant mère, et je vouldroye bien que a elle, selon le fet que se puet comparer d'un chevalier a une dame, la ressemblassez. (p. 1051)

Richarde en remontre ainsi aux hommes, elle incarne l'une de ces femmes à forte personnalité qui, selon J. Batany, accompagnèrent la réaction que l'on pourrait qualifier de féministe à partir de la fin du $\mathrm{XIV}^{\mathrm{e}}$ siècle $^{23}$. Elle prouve que Raison ${ }^{24}$, plus haut dans le livre de

22 Du côté de la chanson de geste, on pourrait penser à Guibourc, prête à défendre Orange avec sept mille compagnes, en l'absence de Guillaume parti chercher des secours (Chanson de Guillaume, éd. F. Suard, Paris, LGF, 2008, v. 2444-51) et qui, dans Aliscans, soutient effectivement, à la tête de ses troupes, le siège d'Orange. Pour ce qui est de la réalité historique, Christine de Pizan affirme en 1405 dans le Livre des trois vertus (II, 9) que les épouses des grands seigneurs doivent être capables de suppléer l'absence de leur mari, y compris dans les affaires militaires : la «dame baronnesse », dit-elle, en plus d'être «saige », doit avoir «si come courage d'omme»; " elle doit avoir cuer d'omme, c'est qu'elle doit savoir des drois d'armes et toutes choses qui y affierent afin que elle soit preste d'ordonner ses hommes se besoings est, et le sache faire pour assaillir et pour deffendre se le cas s'y adonne» (éd. Charity C. Willard, en collab. avec E. Hicks, Paris, Champion, 1989, p. 149-152).

23 Batany 1973 : 66 - qui évoque aussi à ce sujet d'effroyables mégères !

24 Personnage hérité du Roman de la Rose et, à travers lui, de l'Anticlaudianus d'Alain de Lille. Connaissance rectifie l'antiféminisme systématique de Raison en disant 
Thomas de Saluces (chap. 169-173), avait eu tort de dénigrer les femmes.

Entourée de ses «dames et damoiselles » sur le champ de bataille, Richarde n'est pas non plus sans faire penser à la reine des Amazones. Or ces farouches guerrières reparaissent à un autre endroit du Chevalier errant, toujours dans un contexte familial, quoique indirectement. Il est question, dans l'épisode qui se déroule au palais de Fortune, des Neuf Preuses (chap. 181 et 228-235). La liste en est moins stable et moins répandue que celle des Neuf Preux qui lui servit de modèle ${ }^{25}$ mais l'idée en est claire : il s'agit de montrer que certaines femmes peuvent être les égales des hommes, même sur le plan de la vaillance militaire :

De l'autre part en ce palays mesmes estoient les sieges des .ix. damez qui furent de si hault renom come tout le monde scet, car par leurs grans œuvres et merveilleusez furent elles esleuez au monde et comparées aux .ix. preux que dit vous ay, ainsi comme les croniquez le devisent. (chap. 227, p. 751)

Aussi n'est-il guère surprenant de rencontrer, parmi les neuf «élues » de Thomas de Saluces, six Amazones, reines du royaume de «Femenie »: Penthésilée, Synoppe, Hippolyte, Ménalippe, Lampheto, Thomyris ${ }^{26}$. Les trois autres Preuses sont également présentées

qu'« il parloit contre les malez femmez et tricheressez, mais il scet bien, et voir est, que trop noble et digne chose est la femme qui attrait son cuer a bien » (chap. 349, p. 1045). Le même argument resurgira au début du $\mathrm{XV}^{\mathrm{e}}$ siècle dans la querelle du Roman de la Rose.

25 Le motif des Neuf Preux fut lancé vers 1313 par les Vaux du Paon de Jacques de Longuyon; la liste comprend trois héros païens (Hector, Alexandre le Grand, César), trois juifs (Josué, David, Judas Maccabée), trois chrétiens (Arthur, Charlemagne, Godefroy de Bouillon) ; cf. Le Chevalier errant, chap. 227. Les Neuf Preuses apparaissent dans le dernier quart du XIV ${ }^{e}$ siècle, dans le Livre de leesce de Jean Le Fevre et chez Eustache Deschamps; motif repris au Xve par Christine de Pizan (Cité des Dames, Mutacion de Fortune), Martin Le Franc (Champion des Dames). Cf. Finoli 1990 et, pour des perspectives plus larges sur le motif, Schroeder 1971, Cassagnes-Brouquet 2003, Cropp 2002, Van Hemelryck 1998, Mombello 1966, Cerquiglini-Toulet 1993. Au Xv siècle, on considère Bertrand Du Guesclin et Jeanne d'Arc comme le $10^{\mathrm{e}}$ Preux et la $10^{\mathrm{e}}$ Preuse.

26 Christine de Pizan retient les mêmes Amazones dans La Cité des Dames (livre I, chap. 16-19) mais développe nettement leur histoire. Martin Le Franc accorde un 
comme d'illustres guerrières: ce sont Déipylé (femme de Tydée), Sémiramis (reine de Babylone ${ }^{27}$ ) et Theuqua (reine d'Illyrie) ${ }^{28}$.

Le propos relatif à chacune d'entre elles est peu développé : huit à dix-huit vers suffisent à justifier leur statut de femme exceptionnelle (cas particulier de Penthésilée : prose). Mais il est complété, dans le $\mathrm{ms}$. BnF fr. 12559, par deux superbes miniatures de pleine page que Thomas de Saluces a pris soin d'annoncer:

Je vous monstreray par ceste pourtraicture les .ix. preux esleuz, et après les .ix. dames qui en leur compaingnie estoient et leurs armes. (chap. 240, p. $759-60)$

Le terme «pourtraicture », encore rare à l'époque, signe l'avènement de l'art du portrait, qui ne remonte guère qu'à la seconde moitié du $\mathrm{XIV}^{\mathrm{e}}$ siècle. Il est aussi tout à fait exceptionnel dans le texte du Chevalier errant, où toutes les miniatures signalées par le narrateur le sont à l'aide des termes «figure » ou « hystoire ». Dès lors, on est en droit de supposer que Thomas de Saluces songeait à faire représenter à travers ces Preux et Preuses des membres de sa propre famille. À l'aune de cette interprétation, l'expression «les .ix. dames qui en leur compaingnie estoient» gagne en exactitude : les Neuf Preuses ne sont «normalement» pas les compagnes des Neuf Preux, en ce sens qu'elles ne correspondent pas aux femmes qu'on leur connait ; mais si les Preuses sont prétexte à représenter les épouses des marquis de

intérêt particulier à Thomyris et à Penthésilée (Champion des Dames, livre IV, v. 16457-480 et 16513-576, éd. R. Deschaux, Paris, Champion, 1999). Sur Penthésilée, Cassagnes-Brouquet 2004.

27 Elle est souvent invoquée dans la littérature exemplaire du Moyen Âge. Le v. 9504 du Chevalier errant ("Par son senz et par sa vaillance ») reformule à son sujet le couple sapientia-fortitudo. Martin Le Franc s'attarde particulièrement sur son cas (Champion des Dames, livre IV, v. 16409-456).

28 Ces Neuf Preuses ne suivant pas la tripartition des Neuf Preux, une nouvelle série de «femmes vertueuses» (guten Frauen) s'élabora à partir de 1470 (uniquement en Allemagne, alors que le motif des Neuf Preuses était répandu en France et en Angleterre), proposant trois païennes : Lucrèce, Véturie, Virginia ; trois juives: Esther, Judith, Yaël ; trois chrétiennes: sainte Hélène, sainte Brigitte, sainte Elisabeth ; cf. Schroeder 1971 : 173-203, 256-260, 297-299. L'un des intérêts de cette série est d'opérer le lien entre héroïsme féminin et sainteté, trait que nous allons retrouver avec Grisilidis. 
Saluces, on comprend qu'elles sont en leur compagnie du fait du lien conjugal. Enfin, la syntaxe de la phrase d'annonce semble maintenir délibérément une ambiguité : l'expression «et leurs armes » fait-elle seulement référence aux armoiries qui accompagnent chaque Preux et chaque Preuse sur nos deux miniatures, ou bien n'annonce-t-elle pas d'un même élan les armoiries familiales des Saluces, placées juste en dessous de la miniature consacrée aux Preuses ${ }^{29}$ ? Partant, l'assimilation des Preuses et de personnes réelles de la famille des Saluces serait suggérée par la mise en page même, et il n'est pas anodin de relever que cette assimilation privilégie le versant féminin de la famille.

Finalement, le cycle des Neuf Preux et des Neuf Preuses peut être interprété comme une allégorie familiale, revendiquée comme telle. Georges Duby a en effet souligné l'importance des Neuf Preux comme modèle de comportement héroïque pour la classe chevaleresque :

Dans l'existence des hommes et des femmes dont l'œuvre d'art au XIVe siècle avait pour mission d'illustrer les désirs de faste et de bonheur, l'imitation des neuf Preux et des neuf Preuses alternait avec l'imitation de Jésus-Christ. Elle la complétait. Il importait de mimer, dans la vie, les gestes des héros, comme l'on mimait, dans l'attente anxieuse de la mort, ceux du Sauveur ${ }^{30}$.

C'est la raison pour laquelle la représentation des Neuf Preux était, depuis le milieu du XIV siècle, un motif artistique à la mode dans les milieux aristocratiques, tant en France qu'en Italie (enluminures, fresques, tapisseries, vitraux). Les fresques du château de Manta, près de Saluces, confirment cette lecture familiale du motif héroïque. Vers 1420, Valerano, fils illégitime mais bien-aimé de Thomas de Saluces, fit peindre sur les murs de la salle principale de ce château les Preux et Preuses, accompagnés de vers empruntés au roman de son père ${ }^{31}$.

$29 \quad \mathrm{~F}^{\circ} 125 \mathrm{v}$; voir Cabier d'images, figure 3.

30 Duby 1984 : 153.

31 Voir Cabier d'images, figure 4. En Italien, ces fresques sont intitulées «I Prodi e le Eroine »... Un contresens sur le v. 9497 du Chevalier errant a fait prendre le nom «Etioppe » (Éthiopie) pour celui d'une preuse, d'où un décalage entre le texte de Thomas de Saluces et les fresques (qui représentent Ethioppe mais non Ménalippe). 
Or les spécialistes ont reconnu Valerano sous les traits d'Hector de Troie, faisant face à son épouse Clementia Provana, représentée par Penthésilée («qui si vaillant dame fu», précise Thomas de Saluces, p. 732). Le cortège des Preux reconstitue la succession des marquis de Saluces et la continuité de leur pouvoir, pourtant en butte aux vicissitudes de Fortune : Manfred I ${ }^{\text {er }}$ devient Godefroy de Bouillon, Thomas Ire, le roi Arthur, Manfred IV, Judas Maccabée... Et Thomas III (mort en 1416) est transfiguré en Alexandre le Grand! Daniel Arasse peut alors qualifier ce programme iconographique de "galerie dynastique mythique $»^{32}$. Pour d'évidentes raisons de symétrie (non de parité!), les femmes y occupent une place équivalente à celle des hommes; sept d'entre elles sont figurées en armes, tout en gardant les attraits de la beauté féminine.

\section{Grisilidis, l'épouse vertueuse}

De la vaillance martiale incarnée historiquement par Richarde et, de manière mythique, par les Neuf Preuses, tournons-nous vers la vaillance spirituelle de Grisilidis, l'héroïne la plus célèbre de la famille de Saluces. C'est Orose ${ }^{33}$ (autre figure de maitre digne de foi) qui raconte son histoire au Chevalier :

Or vous ay dit, sire chevalier, comment ceste haute Dame Fortune esprouva et troubla ceste vaillante dame Grisilidis, de laquelle bien croy qu'avez oÿ parler et de son grant scens et pacience. (chap. 274, p. 854)

La patience de Grisilidis remplace le courage militaire mais reste, on le verra, un véritable courage, une force d'âme qui cautionne son statut héroïque. Thomas de Saluces avait forcément entendu parler de la fameuse épouse du marquis Gautier de Saluces, qui connut sa

32 Arasse 1989 : 95. De surcroît, ce programme permet au fils illégitime de s'inscrire dans la dynastie officielle des marquis de Saluces: "on pourrait dire que Valerano "s'autofonde" mythiquement dans la lignée familiale, qu'il s'autolégitime en peinture » (Arasse 1989 : 101). Je n’entre pas dans le détail des interprétations, par endroits divergentes, proposées sur ces fresques; outre l'article qui vient d'être cité, cf. D’Ancona 1905, Botta s.d., Fajen 2000, Meneghetti 1989, Piccat 1991.

33 Auteur de l'Histoire contre les païens (415-417) qui servit de relais aux historiographes médiévaux au sujet, entre autres, des Amazones. 
consécration littéraire au XIV siècle avec la nouvelle de Boccace (Décaméron, X, 10) ${ }^{34}$. Celle-ci fut relayée par une version latine de Pétrarque (1374), elle-même traduite en français d'une part par Philippe de Mézières ${ }^{35}$, d'autre part par un anonyme. Thomas de Saluces a combiné ces deux dernières versions en les agrémentant de détails de son cru; il a visiblement pris plaisir à développer l'histoire, qui s'étend sur les chapitres 267 à 274. Quoique légendaire, Grisilidis était alors tenue pour un personnage historique (certaines chroniques attestaient son existence au XI siècle). Rappelons l'histoire :

Gautier, marquis de Saluces, régnait sur ses terres sans se soucier de sa postérité. Ses sujets le conjurent de prendre femme. Il accepte mais choisit, à la surprise générale, Grisilidis, la fille d'un humble paysan. Grisilidis promet entière soumission au marquis et s'attire par ses vertus l'amour de tous ses sujets. Mais lorsqu'elle met au monde une fille, Gautier décide d'éprouver la constance de sa femme sous prétexte qu'on commence à jaser sur sa vile origine, et prétend faire disparaître l'enfant. Bien que désespérée, Grisilidis se plie à la volonté de son seigneur (en réalité, Gautier a fait mener secrètement sa fille chez une parente). Quatre ans plus tard, Grisilidis accouche d'un fils et Gautier renouvelle l'épreuve. Plus tard encore, il va jusqu'à prétendre répudier Grisilidis et prendre une épouse plus digne de son rang. Grisilidis accepte tout, même de venir aider au service lors des noces de Gautier. Entretemps ce dernier a fait revenir incognito ses enfants, âgés de douze et huit ans. Il laisse croire qu'il va épouser la jeune demoiselle, jusqu'au moment où il rend publiquement hommage à la patience de Grisilidis, la reconnaissant comme son épouse chérie. Il rend les deux enfants à l'amour de leur mère et la fête se conclut par des réjouissances générales. Gautier et Grisilidis vécurent encore vingt ans et leurs enfants prospérèrent.

D’entrée de jeu, le nécessaire mariage de Gautier engage la question éminemment politique de la succession et de la légitimité du pouvoir à transmettre (préoccupation récurrente, on l'a vu, de

34 Golenistcheff-Koutousoff 1933, Morse 1985, Brownlee 1992, Lefèvre 1992. Parmi de nombreux admirateurs et admiratrices, Christine de Pizan n'a pas manqué d'intégrer Grisélidis, dont elle souligne la force de caractère, à sa Cité des Dames (livre II, chap. 50). Cf. L'Histoire de Griselda 2000-2001 (t. 1 : Prose et poésie, XIVe-XVI es.; t. 2 : Théâtre XVII s.) La fortune littéraire de Grisélidis, propagée par la Bibliothèque bleue, se prolonge jusqu’̀ Perrault.

35 L'histoire est insérée dans le Livre de la vertu du sacrement de mariage et du réconfort des dames mariées (entre 1385 et 1389). 
Thomas de Saluces dans Le Chevalier errant). Dès lors, son union avec la simple paysanne qu'est Grisilidis semble une folie. Mais il n'en est rien grâce à la haute vertu de Grisilidis, en quelque sorte anoblie par des qualités qui ne sont pas d'ordre temporel mais spirituel :

Ycellui Janicole avoit une fille appellée Grisilidiz, belle de toutez faitures de corps et de membres, et meilleure en bonnez meurs, de vérité raemplie tant que plus ne pouoit par la grâce de Dieu. Et avoit esté nourrie en grant povrecté, et ne sçavoit que estoit aise, car elle n'en avoit rien appris, mais toutefois son courage estoit meur en virginité ; $[\ldots]$ et estoit en elle toute obéissance de pitié et de tout bien, et en parfaicte virginité. (chap. 268, p. 833)

C'est pourquoi son histoire exemplaire n'intéresse pas que la famille de Saluces mais se présente comme le "mirouer des dames mariées » (titre du chapitre 267, emprunté à Mézières) ${ }^{36}$. La « haulte et merveilleuse vertu de pacience, obedience, vraye humilité et constance » annoncée dans la suite du titre du chapitre 267 se vérifie au fil des péripéties du récit, régulièrement ponctué par les termes "pacience» et «constance». Lorsque, apparemment déchue de tous ses droits, Grisilidis veille, en simple servante, aux préparatifs du supposé remariage de Gautier (chap. 273), elle incarne jusqu'au sublime la fonction traditionnelle qui fait de la femme l'intendante de l'espace domestique (par opposition à l'ordre masculin, voué à l'espace public). Les sujets du marquis entrevoient bientôt derrière la réalité conjugale une intention divine :

Pluseurs cuidoient que elle [Grisilidis] feust envoiée des Cieulz au salut du commun et bien publique. (chap. 269, p. 838)

Le subterfuge du marquis de Saluces, pour cruel qu'il paraisse, démontre qu'il faut savoir dépasser les apparences (comme lui-même avait su discerner dans l'humble paysanne la seule épouse digne de lui). De fait, les lecteurs sont à leur tour invités à imiter en bons chrétiens l'exemple de Grisilidis pour savoir rester fermes dans l'adversité, comme l'explique la conclusion édifiante (textuellement empruntée elle aussi à Mézières) qui fait de l'histoire une parabole de type évangélique dont le message vaut pour l'humanité entière :

36 Andries 1981 signale que "certains livrets bleus du XVIII" siècle font paraitre à la suite de Griselidis un court texte sur L'Amour conjugal des femmes illustres » (p. 220). 
Et est assavoir que ceste histoire a esté escripte en la memoire des honneurs ${ }^{37}$, et non tant seulement afin que les matrosnez et dames de nostre temps doient ensuir la pacience de ceste noble dame, laquelle pacience semble aussi comme impossible a porter. C'est assavoir que ce que elle fist a son mari de la vertu de pacience, eulz le vueillent faire a Dieu, combien que Dieu ne tempte pas les gens, selon ce que dist saint Jaques l'Appostre, comme le fist le marquis son espouse; mais aucune foys Dieu les veult esprouver, et conssent que nous ayons souvent adversité par mainte tribulacion, non pas pour cognoistre notre courage comme fist le marquis. Car avant que nous feussions oncques néz, il savoit clerement que nous devions estre, mais il sueffre a ce que par les tribulacions continuellez nostre propre fragilité nous soit monstrée et de nous bien congneue. (chap. 274, p. 853-54).

Avatar féminin de Job, Grisilidis se distingue de celui-ci par sa soumission immédiate à toutes les épreuves, sans l'ombre d'une plainte $^{38}$. On discerne aussi l'idée paulinienne selon laquelle la faiblesse humaine, investie par la confiance en Dieu, peut se transmuer en force ${ }^{39}$. Au rebours de son sens premier, l'histoire de Grisilidis n'a finalement pas pour vocation principale d'imposer une image subalterne de la femme ${ }^{40}$. La "pacience » de l'épouse qui, d'un point de vue réaliste, paraît au narrateur «impossible a porter», appelle l'interprétation allégorique. La «vaillance» morale dont fait preuve Grisilidis par sa douce abnégation fait d'elle tout à la fois une sorte de sainte domestique et un avatar féminin du type du miles Christi. Grisilidis était d'ailleurs considérée comme une

37 Golenistcheff-Koutousoff 1933 signale l'expression « escripte en la memoire des honneurs » comme une leçon fautive caractéristique d'un groupe de manuscrits de l'histoire de Grisilidis (p. 135). Toutefois l'expression peut faire sens dans ce contexte d'honneur familial dont Thomas de Saluces exalte la mémoire, d'autant que l'expression reparait ailleurs : «je mis en memoire les honneurs qui la leur furent donnés » (chap. 152, p. 639).

38 Duby \& Perrot 1991: 466.

39 Cf. II Co 12, 9-10.

40 Même l'auteur du Mesnagier de Paris (traité d'éducation domestique de 1393), qui a inclus l'histoire de Grisilidis dans la section consacrée au devoir d'obéissance, manifeste son malaise face à la cruauté du marquis de Saluces et se défend de vouloir intimider sa jeune épouse en qui il a toute confiance (cf. éd. G.E. Brereton \& J.M. Ferrier, Paris, LGF, 1994, p. 232). 
«bienheureuse » (beata) en France et en Italie ${ }^{41}$. La version de Thomas de Saluces rejoint bien une préoccupation exemplaire typique du traitement de l'histoire de Grisilidis aux XIVe-XVe siècles. Dans cette optique chrétienne, sa vie devient une leçon pour tous les hommes, sans distinction de sexe désormais. De fait, Thomas de Saluces dut chercher durant sa captivité quelque réconfort dans la méditation de son histoire ${ }^{42}$.

En définitive, Richarde et Grisilidis incarnent deux facettes contrastées de ce que peut être une héroïne à la fin du XIVe siècle. Faute du mot "héroïne », la notion d'hérö̈sme féminin s'exprime dans le terme "vaillance » et ses dérivés, récurrents dans les chapitres évoqués du Chevalier errant et tout aussi bien applicables aux hommes. Force active, guerrière ou force passive, patiente, ces deux actualisations de la "vaillance» se rejoignent dans l'une des quatre vertus cardinales, le courage (fortitudo, que Thomas de Saluces nomme «forteresce» et allégorise comme l'un des enfants de Dame Connaissance au chapitre 378$)^{43}$.

Toute héroïne n'a pas à récupérer les attributs physiques de l'héroïsme masculin ; le christianisme a promu, à travers la figure de la sainte (et ses avatars), une autre forme d'héroïsme féminin. Si les héroïnes (historiques comme légendaires) de Thomas de Saluces sont dépourvues de la virginité qui caractérise ordinairement l'héroïne ${ }^{44}$,

41 Cf. Golenistcheff-Koutousoff 1933 : 133.

42 Signalons enfin la «suite rétroactive » que constitue l'histoire de Guillaume de Saluces, père de Gautier (chap. 335, apport original de Thomas de Saluces); Grisilidis est aussi celle qui rédime la tromperie qu'eut à subir de sa femme l'infortuné Guillaume. De la littérature lignagère comme moyen de régler les névroses familiales...

43 «Forteresce est force de courage qui efforce le pouoir de l'ame espirituelle. Et Forteresce est efforcement de arme par laquelle est vivifiée Force corporelle, et Force est noblece et sainteté de courage » (p. 1123).

44 L'héroïne est habituellement vierge ou en partie désexuée, au point qu'on s'interroge sur ce qui reste en elle de féminin (cf. le cas de Jeanne d'Arc). «D'après saint Ambroise, dont le De Virginibus est un texte de référence fondamental, la vierge peut devenir virago, une femme "virile" par l'appropriation 
c'est parce que, s'inscrivant dans un projet idéologique d'ordre familial, elles ont à remplir cette fonction essentielle qu'est la perpétuation de la lignée. Sur leur destinée plane en outre l'ombre, fugitive ou plus pesante, de Fortune, comme sur celle de Thomas de Saluces. Le Chevalier n'est le héros du livre du marquis de Saluces qu'en tant que personnage principal mais il ne se signale guère par des hauts faits. Le Chevalier errant n'est certes pas un roman "féministe » (Thomas fait la part des «bonnes » et des « mauvaises femmes ») mais il semble que lorsque l'héroïsme masculin est en crise (la captivité de Thomas III et la fragilité de son pouvoir sur le marquisat de Saluces en sont l'indice), l'héroïsme féminin puisse, au moins fantasmatiquement et temporairement, jouer en littérature un rôle compensateur.

\section{Bibliographie}

AnCona Paolo D', 1905, « Gli affreschi del castello di Manta nel Saluzzese », L'Arte, 8, p. 94-106 et 183-198.

ANDries Lise, 1981, Moyen Áge et colportage. Robert le Diable et autres récits, Paris, Stock.

ARASSE Daniel, 1989, «Portrait, mémoire familiale et liturgie dynastique : ValeranoHector au château deManta », in Augusto GENTILI (dir.), Il Ritratto e la Memoria. Materiali I, Rome, Bulzoni, p. 93-112.

AriÈs Philippe \& Georges Duby (dir.), 1985, Histoire de la vie privée, 2. De l'Europe féodale à la Renaissance, Paris, Seuil.

Batany Jean, 1973, Approches du "Roman de la Rose", Paris, Bordas.

BотTA L., s.d., Il castello della Manta, Turin.

BoucheT Florence, 1995, Lire, voir, écrire an XIVe siècle : étude du Livre du Chevalier errant de Thomas de Saluces, Université de Paris IV-Sorbonne.

—, 2001, «La nouvelle à l'épreuve du roman médiéval : le Livre du Chevalier errant de Thomas de Saluces », in Vincent Engel \& Michel Guissard (dir.), La Nouvelle de langue francaise aux frontières des autres genres, duMoyen Agge à nos jours, Louvain-laNeuve, Academia Bruylant, p. 7-22.

des qualités spirituelles normalement réservées aux hommes» (Ueltschi 1993: 28). 
BROWNLEE Kevin, 1992, "Commentary and the Rhetoric of Exemplarity : Griseldis in Petrarch, Philippe de Mézières, and the Estoire», The South Atlantic Quarterly, $91 / 4$, p. $865-890$.

ButAud Germain \& Valérie PIÉTRI, 2006, Les enjeux de la généalogie, XIIe-XVIIIe siècle. Pouvoir et identité, Paris, Éditions Autrement.

Cassagnes-Brouquet Sophie, 2003, «Les Neuf Preuses, l'invention d'un nouveau thème iconographique dans le contexte de la guerre de Cent Ans ", in L. Capdevilla, S. Cassagnes, M. Cocaud, D. Godineau, F. Rouquet \& J. Sainclivier (dir.), Le Genre face aux mutations, Masculin et féminin du Moyen Âge à nos jours, Rennes, PUR, p. 279-290.

—, 2004, "Penthésilée, reine des Amazones et Preuse: une image de la femme guerrière à la fin du Moyen Âge », CLIO Histoire, Femmes et Sociétés, 20, p. 169-180.

Cazalé Bérard Claude \& Christiane Klapisch-Zuber, 2004, "Mémoire de soi et des autres dans les livres de famille italiens », Annales. Histoire, Sciences sociales, $59 e$ année, 4, p. 805-826.

Centlivres Pierre, Fabre Daniel \& Françoise Zonabend (dir.), 1998, La Fabrique des Héros, Paris, Publications de la Maison des Sciences de l'Homme.

Cerquiglini-Toulet Jacqueline, 1993, «Fama et les Preux : nom et prénom à la fin du Moyen Âge », Médiévales, 24, p. 35-44.

Cropp Glinnis M., 2002, « Les vers sur les neuf Preux », Romania, 120, p. 449-482.

DuBy Georges, 1984, Le Moyen Âge. Fondements d'un nouvel humanisme, 1280-1440, Genève, Skira.

—, 1988, "Remarques sur la littérature généalogique en France aux XIe et XII ${ }^{\mathrm{e}}$ siècles », La société chevaleresque, Paris, Flammarion, p. 167-180.

Duby Georges \& Michelle Perrot (dir.), 1991, Histoire des femmes en Occident. Tome 2,Le Moyen Âge, Paris, Plon.

FAJEN Robert, 2000, «Malinconia di un lignaggio. Lo Chevalier errant nel castello della Manta », Romania, 118, p. 105-137.

-, 2003, Die Lanze und die Feder. Untersuchungen zum "Livre du Chevalier errant» von Thomas III, Markgraf von Saluzzo, Wiesbaden, Reichert.

FAliu Odile \& Marc Tourret (dir.), 2007, Héros d'Achille à Zidane, Paris, Bibliothèque nationale de France.

FinOli Anna Maria, 1990, "Le donne, e’ cavalier...” : il topos dei Nove Prodi e delle Nove Eroine nel Chevalier errant di Tommaso III di Saluzzo ", Il Confronto letterario, 13, p. 109-122.

GolenistchefF-KoutousofF Elie, 1933, L'Histoire de Griseldis en France an XIV et au XVe siècle, Genève, Droz. 
GueNÉE Bernard, 1976-1977, «Temps de l'histoire et temps de la mémoire au Moyen Âge ", Annuaire-Bulletin de la Société de l'Histoire de France, p. 25-35.

LeFÈvre Sylvie, 1992, "Griseldis », in Geneviève HASENOHR \& Michel ZINK (dir.), Dictionnaire des Lettres françaises. Le Moyen Age, Paris, LGF, p. 581.

MeNeGHeTti Maria Luisa, 1989, « Il manoscritto fr. 146 della Bibliothèque Nationale di Parigi, Tommaso di Saluzzo e gli affreschi della Manta », Romania, 110, p. 511535.

Mombello Giani, 1966, «Les Complaintes des .ix. malheureux et des ix. malheureuses. Variations sur le thème des Neuf Preux et du Vado mori », Romania, 87, p. 345-378.

Mordenti Raul, 2004, "Les livres de famille en Italie », Annales. Histoire, Sciences sociales, $59^{\mathrm{e}}$ année, 4 , p. $785-804$.

Morse Charlotte C., 1985, «The exemplary Griselda », Studies in the Age of Chaucer, 7, p. 51-86.

NARdone Jean-Luc \& Henri Lamarque (dir.), 2000-2001, L'Histoire de Griselda. Une femme exemplaire dans les littératures européennes, Toulouse, PUM, 2 vol.

Pastoureau Michel, 1986, Figures et couleurs. Études sur la symbolique et la sensibilité médievvales, Paris, Le Léopard d'Or.

Piccat Marco, 1991, «Le scritte in volgare dei Prodi e delle Eroine della sala affrescata nel castello di La Manta », Studi Piemontesi, 20/1, p. 141-166.

SCHROEDer Horst, 1971, Der Topos der Nine Worthies in Literatur und bildender Kunst, Göttingen, Vandenhoeck \& Ruprecht.

Ueltschi Karin, 1993, La Didactique de la chair. Approches et enjeux d'un discours en français au Moyen Âge, Genève, Droz.

Van HemelrycK Tania, 1998, «Où sont les "Neuf Preux "? Variations sur un thème médiéval », Studi francesi, XLII-1, p. 1-9.

WARD Marvin James, 1984, A critical edition of Thomas III, marquis of Saluzzo's Le Livre du Chevalier errant (French Text), Ph.D., The University of North Carolina at Chapel Hill, 3 vol. 